\title{
DEMANDAS DESDE LA EXCLUSIÓN: REPRESENTATIVIDAD DEMOCRÁTICA Y CUOTAS DE PARTICIPACIÓN POLÍTICA EN COSTA RICA
}

\section{DEMANDS FROM EXCLUSION: DEMOCRATIC REPRESENTATION AND GENDER QUOTAS IN COSTA RICA}

\section{Montserrat Sagot Rodríguez*}

\section{RESUMEN}

\begin{abstract}
En este artículo se analiza la aplicación del sistema de cuotas de participación política en Costa Rica como mecanismo de acción afirmativa para las mujeres. Se analiza además la evolución de la legislación sobre cuotas en el país, la cual culminó con la aprobación del nuevo Código Electoral, que estableció la paridad de género. Asimismo, se discute sobre la efectividad de cada tipo de normativa para incrementar la representación descriptiva de las mujeres, así como el impacto del sistema de cuotas en el avance de una agenda feminista de igualdad y justicia de género.
\end{abstract}

PALABRAS CLAVE: COSTA RICA * DEMOCRACIA * CUOTAS DE PARTICIPACIÓN POLÍTICA * IGUALDAD DE GÉNERO * FEMINISMO

\section{SUMMARY}

This article analyzes the implementation of the gender quota system in Costa Rica as an affirmative action mechanism for women. It also analyses the evolution of the quota legislation in the country, which culminated with the passing of a new Electoral Code that established gender parity. The article also discusses the effectiveness of each type of legislation to increase the descriptive representation of women, as well as the impact of the quota system on the advancement of a feminist agenda of gender equality and justice.

KEY WORDS: COSTA RICA * DEMOCRACY * GENDER QUOTAS * GENDER EQUALITY * FEMINISM 
LAS MUJERES Y LA REPRESENTACIÓN POLÍTICA: UNA HISTORIA DE EXCLUSIÓN

La larga historia de debate y acciones para construir una sociedad democrática han procedido por siglos sin tomar en consideración a las mujeres y a otros grupos subordinados. Lo anterior porque las diferentes teorías sobre la democracia - desde los griegos hasta el presente-y las prácticas políticas tradicionales siempre han asumido la existencia de una ciudadanía abstracta, sin cuerpo, sin clase, sin etnia y sin género. Como lo plantea Anne Phillips (1996), la relación entre democracia e igualdad es un asunto reciente. Desde sus inicios como movimiento social en la Europa del siglo XVIII, el feminismo ha tratado de establecer esa conexión y de introducirla en las nuevas discusiones sobre la democracia que se inician con la Ilustración, al plantear que una genuina igualdad entre mujeres $y$ hombres solo puede ser alcanzada si se reconocen las diferencias de género y su impacto en la participación política y en el acceso a la representatividad democrática de cada uno de los sexos.

En diferentes momentos de los siglos XVII y XVIII, autoras como Mary Astell y Mary Wollstonecraft plantearon esa problemática cuando se preguntaban cómo aquellos que rechazaban la noción de la soberanía absoluta del Rey, la aceptaban como natural cuando se trataba de la soberanía de un marido en su casa; o cuando no cuestionaban el hecho de que las mujeres no tuvieran representación en las deliberaciones sobre el destino de sus gobiernos y sociedades (Freedman, 2007). Concluían así estas autoras $-y$ muchas otras en los siglos venideros- que la transición a una sociedad verdaderamente democrática solo puede ocurrir cuando las mujeres se conviertan en participantes activas en la vida política, dado que la igualdad es incompatible con la subordinación.

A pesar de no haber sido invitadas a estos debates, las feministas, junto con teóricos de otras corrientes de pensamiento emancipadoras, han planteado serias críticas al modelo de la democracia liberal representativa. Autoras como Carole Pateman (1995), Anne Phillips (2002), Iris Marion Young (2000), Susan Moller
Okin (1989) y otras, han argumentado que el sistema de la democracia formal les niega a las mayorías la posibilidad de participar en la toma de decisiones. Es decir, en el mejor de los casos, se le concede a la ciudadanía el derecho de ejercer el sufragio para escoger a los gobernantes, pero luego se les excluye de los procedimientos del control democrático, de la representación popular y de la participación directa.

Desde esa perspectiva, la pretendida igualdad política de un sistema democrático formal muestra su inconsistencia en esta organización social que excluye a las mayorías de la posibilidad de ser electos o electas y consecuentemente de participar en la toma de decisiones. Así, la democracia formal muestra una verdadera disonancia entre la ciudadanía y sus representantes, dado que, en la mayoría de los casos, esos representantes no reflejan la diversidad que caracteriza a la población, en la medida en que muchos sectores no tienen acceso a la representación democrática o tienen un acceso muy restringido a esta.

Como resultado de que las mujeres están entre las grandes excluidas de estos procesos, el movimiento feminista ha luchado desde sus orígenes por revertir esta situación. A partir de 1779, el naciente movimiento feminista definió como una de sus prioridades políticas la lucha por extender a las mujeres aquellos derechos igualitarios, concebidos bajos las nuevas condiciones sociales, como derechos "naturales" de los hombres. Es así, como respondiendo a la ola revolucionaria de la modernidad, las primeras feministas intentaron universalizar unas reglas sociales que habían sido formuladas originalmente para una población limitada y particular: los hombres.

Los argumentos empleados por estas feministas se basaban en la concepción de una humanidad común y unas características esenciales compartidas por todos los seres humanos, independientemente de su sexo. Se planteaba así, que más allá de la especificidad de las condiciones sociales y culturales (en las que las mujeres ocupaban una posición desigual) existían unos ideales trascendentales de racionalidad y justicia que deberían aplicarse universalmente (Sagot, 1997). Con el desarrollo de 
estas ideas sobre la igualdad universal se inicia también la lucha por una serie de reivindicaciones concretas sobre el acceso de las mujeres a los recursos económicos, a la educación, al poder político $y$, en general, a todas las esferas de toma de decisiones. De esta forma, la aspiración de un sector importante de las feministas, en particular las de raíz liberal, por alcanzar el reconocimiento de una igualdad esencialmente humana - por medio de la extensión y reconocimiento de derechos a las mujeres - se había iniciado y se mantendrá, con algunas variantes, hasta nuestros días.

En las últimas décadas, los esfuerzos de las mujeres por trascender su papel meramente reproductivo en los procesos sociales y políticos $y$ por ocupar espacios en las esferas del tradicional poder masculino, empezaron a rendir sus frutos. De una masa amorfa políticamente, las mujeres pasan a ser un nuevo sujeto social - heterogéneo y a veces hasta contradictoriopero que ha superado la etapa de la denuncia $y$ ha llegado al diseño de estrategias y propuestas para incidir en las agendas públicas y para ampliar la ciudadanía de las mujeres.

\section{LAS CUOTAS COMO MECANISMOS DE DIFERENCIACIÓN PARA LA IGUALDAD}

Una de las estrategias desarrolladas y propuestas, como medida temporal y compensatoria, para alcanzar los objetivos de la igualdad de género $y$ de la representatividad democrática es el sistema de cuotas de participación política. Este mecanismo es entendido como un instrumento para resolver la tensión de un sistema que pretende ser igualitario (la democracia formal liberal), pero que genera grandes desigualdades y exclusiones, sobre todo cuando está aparejado a un sistema económico capitalista, cuya esencia es justamente la desigualdad. En ese sentido, si bien las constituciones de los países democráticos del mundo garantizan la igualdad formal, esta es una condición necesaria, pero no suficiente para alcanzar la paridad y la equidad. De hecho, la igualdad desde el punto de vista de la distribución de recursos, de las cargas y beneficios entre los miembros de una sociedad, más que una realidad, continúa siendo un ideal, una aspiración (Camacho; Lara y Serrano, 1997). Como lo plantea Diana Maffía:

Porque desde el comienzo de la democracia, libres e iguales eran sólo los varones, adultos, blancos y propietarios. Ni las mujeres, ni los indígenas, ni los africanos esclavizados, ni los niños eran considerados ciudadanos. Tampoco los pobres, porque la condición primera de la ciudadanía era tener propiedad privada. Muchos de los sujetos entonces excluidos todavía siguen reclamando sus derechos, porque lo que el lenguaje promete como universal, la realidad no lo cumple (Maffía, 2004: 3).

En este contexto, surgen las llamadas políticas de diferenciación para la igualdad o políticas de acción afirmativa, las que explícitamente tratan desigualmente a quienes en la práctica son desiguales, con el fin de disminuir las distancias económicas, sociales, culturales y políticas entre los miembros de una sociedad (Camacho; Lara y Serrano, 1997). Como parte de estas políticas, los sistemas de cuotas pretenden desarrollar mecanismos representativos que reconozcan explícitamente la diferencia y la desigualdad de género $y$, de este modo, garantizar una nueva proporcionalidad entre los sexos en aquellos ámbitos donde se toman las decisiones políticas. En ese sentido, los sistemas de cuotas de participación política no tratan a las mujeres como "incapaces" o necesitadas de ayuda, sino que reconocen la existencia de un orden social desigual, que las ha excluido de forma deliberada de los espacios de toma de decisiones. Desde la anterior perspectiva, como lo expresa Marcela Ríos:

Las cuotas permiten que el esfuerzo de acceder a los cargos de representación política no resida exclusivamente en las mujeres (en forma individual), sino en quienes controlan el proceso de selección. De esta forma, las cuotas de género se enfocan $y$ entienden como una responsabilidad colectiva a un derecho individual (Ríos, 2008: 15). 
El sistema de cuotas, por medio de mecanismos institucionalizados, busca así asegurar que las mujeres constituyan, al menos, una "minoría decisiva" del 30\% al 40\% como medida temporal mientras que se eliminan las barreras históricas que impiden su acceso a los puestos de decisión política (Dahlerup, 1998). Este mecanismo permite a las democracias formales corregir de algún modo el desequilibrio forjado por siglos de opresión y exclusión de las mujeres (Phillips, 1996). Ello supone el reconocimiento de que las mujeres no se encuentran en el mismo piso que los varones; es decir, que existen diferencias en su situación y condición, que las colocan en una posición de desventaja para participar en las actividades del ámbito público, dominio masculino por excelencia. Desde esa perspectiva, las cuotas no son concesiones gratuitas para las mujeres, sino medidas compensatorias frente a la discriminación, a la negación de ciudadanía y a la exclusión social.

Si bien las políticas de diferenciación para la igualdad no se limitan a los sistemas de cuotas de participación política e incluyen también otros mecanismos - como los sistemas impositivos diferenciados, donde los ricos se supone que pagan más y los pobres menos, o las becas para estudiantes de grupos pobres o históricamente discriminados o los mismos programas sociales focalizados, que privilegian a los más desfavorecidos-, es cuando se llega al terreno de las acciones afirmativas para mujeres que muchas veces se han levantado en contra, alegando discriminación contra los hombres, concesiones injustificadas para las mujeres, contradicciones con el principio de igualdad y negación de los principios de la representación democrática. Pero esto siempre ha ocurrido cuando las mujeres demandan justicia e igualdad.

Según Rosalía Camacho, Silvia Lara y Ester Serrano (1997), el proceso para llegar a plantear acciones afirmativas, llevado adelante por el movimiento de mujeres, tuvo varias etapas. En un primer momento de la lucha, la estrategia consistió en demandar la abolición de las leyes sexistas que explícitamente excluían a las mujeres. Sin embargo, la progresiva eliminación de las restricciones que las leyes imponían a las mujeres, no condujo a una igualdad sustantiva entre mujeres $y$ hombres, como lo comprobaron las sufragistas y lo siguen comprobando las mujeres $y$ las personas que pertenecen a otros grupos excluidos, aun en pleno siglo XXI.

Un segundo momento consistió en demandar leyes que expresamente combatieran y prohibieran la discriminación por género. Sin embargo, tampoco estas disposiciones surtieron el efecto deseado. Se concluyó entonces que no era suficiente prohibir la discriminación sexual para alcanzar la equidad.

En la tercera etapa, algunos sectores del movimiento feminista plantean que es imperativo adoptar medidas especiales que aseguren en la práctica $y$ de forma efectiva la igualdad de oportunidades. Durante esta etapa, se consiguió un respaldo importante por medio de instrumentos internacionales, como la Convención para la eliminación de todas las formas de discriminación contra la mujer (CEDAW, 1979), que en su artículo 4 establece que: "las medidas especiales de carácter temporal encaminadas a acelerar la igualdad de facto entre el hombre y la mujer no serán consideradas discriminatorias, siempre que sean temporales".

Se inicia así un proceso de lucha política por tratar de establecer medidas concretas que permitieran contrarrestar o corregir la discriminación contra las mujeres, entendiendo que las reglas supuestamente neutrales de la democracia liberal habían conducido a resultados desiguales. Se habla entonces de la necesidad de desarrollar medidas de justicia compensatoria y de justicia redistributiva frente a la supuesta justicia ciega, que solo había resultado ciega para ver la discriminación contra las mujeres y contra otros grupos excluidos.

El sistema de cuotas de participación política, en particular lo que se conoce como leyes electorales de cuotas, emergió por primera vez en Noruega en los años 70, donde la proporción de mujeres en los puestos de elección popular, especialmente en el parlamento, siempre ha estado entre las más altas del mundo (Caul, 2001). Desde ese momento, cerca de 50 naciones han implementado algún tipo de 
leyes de cuotas, sobre todo para las elecciones parlamentarias. Asimismo, en cerca de 70 países, diversos partidos políticos han adoptado de forma voluntaria mecanismos de cuotas, auto imponiéndose obligaciones sobre la composición de género de sus órganos directivos y para las listas de candidaturas que presentan para cargos de elección popular (Ríos y Villar, 2006).

Los sistemas de cuotas implican el reconocimiento social y explícito de una serie de situaciones que han impedido el acceso paritario de las mujeres a los puestos de decisión política, entre otras cosas:

1. El reconocimiento de la existencia de la discriminación contra las mujeres tanto en el ámbito público como privado.

2. El reconocimiento de la existencia de asimetrías en la ubicación de mujeres y hombres, $y$ en sus posibilidades de acceso a los espacios de toma de decisiones.

3. El reconocimiento de que la discriminación y las asimetrías no se van a erradicar únicamente con enunciados formales o con declaraciones abstractas de igualdad, como las contenidas en las constituciones políticas.

4. El reconocimiento de que las reglas $y$ principios neutrales frente al género conducen a resultados desiguales.

5. La voluntad de superar la discriminación, como un medio para construir una sociedad verdaderamente democrática y equitativa (Camacho; Lara y Serrano, 1997).

Si bien, este sistema enfrenta serias críticas, tanto de los sectores más conservadores como de las mismas feministas, en los países donde se ha implementado, este ha demostrado ser un buen mecanismo para promover la llegada de mujeres a los puestos de elección popular. De hecho, los sistemas de cuotas han incrementado sustancialmente el número de mujeres en puestos de toma de decisiones en todos los lugares donde se aplica correctamente. Además, es el único mecanismo que puede ser regulado jurídicamente, comprobado, evaluado y medido, y cuya aplicación es concreta (Torres,
2001). Asimismo, a corto plazo pueden verse los resultados de su aplicación y medirse cuantitativamente el avance de las mujeres, sobre todo en términos de su representación descriptiva ${ }^{1}$.

\section{EVOLUCIÓN DEL SISTEMA DE CUOTAS EN COSTA RICA}

Costa Rica es el único país en el mundo que ha utilizado tres tipos diferentes de legislación sobre cuotas en un período relativamente corto (Jones, 2004), lo que lo convierte en un escenario único para analizar la efectividad de los diferentes tipos de legislación: cuota voluntaria, cuota sin obligatoriedad de colocar a las mujeres en puestos elegibles y cuota con obligatoriedad de colocar a las mujeres en puestos elegibles. Asimismo, Costa Rica es uno de los pocos países en el mundo que ha aprobado una legislación estableciendo la paridad y la alternabilidad por sexo en las papeletas de elección popular. Desde esa perspectiva, en las siguientes páginas se analizan los procesos sociales y políticos que influenciaron la adopción de las diferentes modalidades del sistema de cuotas y que culminaron con la aprobación de la normativa que estableció la paridad de género. En el artículo también se analiza la efectividad de cada tipo de normativa para incrementar la representación descriptiva de las mujeres, así como el impacto del sistema de cuotas en el avance de una agenda feminista de igualdad $y$ justicia de género.

Los primeros intentos para plasmar en la legislación nacional algunas normas tendientes a establecer la aplicación de una cuota mínima de participación política para las mujeres se dieron en 1988. En ese momento, después de una fuerte presión del movimiento de mujeres, la Primera Dama de ese entonces, Margarita Penón, negoció la presentación ante la Asamblea Legislativa de un proyecto para la promulgación de una ley de igualdad real, con el objetivo de ayudar a trascender la igualdad formal, tal y como está contemplada y definida

\footnotetext{
1 Por representación descriptiva se entiende la presencia de miembros de un determinado grupo social en un ente político (Franceschet, 2008).
} 
en las leyes y en la Constitución. Inspirado en la Convención para la Eliminación de Todas las Formas de Discriminación contra la Mujer (CEDAW: The Convention on the Elimination of All Forms of Discrimination against Women), este proyecto de ley fue en lo fundamental redactado por abogadas $y$ activistas feministas, quienes se comprometieron a trabajar en conjunto con la Primera Dama con el fin de enviar al Congreso un proyecto de ley para promover una verdadera igualdad de las mujeres en diferentes ámbitos, incluyendo el de la representación política.

El proyecto de ley original contenía varias disposiciones para establecer un sistema de cuotas. Sin embargo, las fuertes reacciones negativas que se provocaron en la Asamblea Legislativa durante su debate motivaron su exclusión del texto final que fue aprobado en 1990 con el nombre de "Ley de Promoción de la Igualdad Social de la Mujer"2. Como puede derivarse del título con el que finalmente fue aprobada esta ley, los contenidos originales que pretendían trascender el concepto de igualdad formal fueron eliminados, incluyendo la propuesta de establecer cuotas obligatorias de representación política para las mujeres.

Si bien, la mención explícita a las cuotas se eliminó de la Ley, se incluyó un capítulo de Derechos Políticos de las Mujeres, que hacía referencia a la obligación de los partidos políticos de incorporar en sus estatutos mecanismos eficaces para promover $y$ asegurar la participación efectiva de las mujeres en porcentajes significativos (artículos 5 y 6 del Capítulo II de la Ley 7142). Sin embargo, dado que este capítulo fue redactado con términos imprecisos y vagos como "mecanismos

2 Entre los argumentos esgrimidos en contra del proyecto de ley de igualdad real se planteó que la proporcionalidad de género en la representación política era inconstitucional, que el proyecto era innecesario ya que no había discriminación contra las mujeres en Costa Rica, que reconocer la necesidad de una reforma de esa naturaleza sería aceptar que tanto la Constitución como las leyes discriminaban por razones de género, lo cual no era cierto, que no se puede imponer la igualdad por la fuerza $y$, finalmente, que las promotoras del proyecto de ley eran "mujeres frustradas" (Badilla, 1997). eficaces" y "porcentajes significativos" no llevó a ningún resultado concreto. En ese sentido, si bien varios partidos políticos realizaron algunas reformas a sus estatutos en el año 1992, la vaguedad de la ley hizo que no se establecieran mecanismos precisos de aplicación de las cuotas. En resumen, en este período se produjeron reformas de buenas intenciones $y$ declaraciones de buena voluntad, pero sin ningún impacto práctico para las mujeres, lo que demostró que en la mayoría de los casos, la cuota voluntaria solo tiene efectos declarativos que fácilmente se quedan en el papel.

Partiendo de que lo dispuesto en la Ley de Promoción de la Igualdad Social de la Mujer no se estaba cumpliendo, también en 1992, la diputada del Partido Liberación Nacional, Gladys Rojas, presentó ante la Asamblea Legislativa un proyecto de reforma al Código Electoral con el fin de introducir como obligatorio el sistema de cuotas de participación de las mujeres en los partidos políticos. En esta ocasión, el Tribunal Supremo de Elecciones (TSE) se pronunció en contra de la propuesta, alegando que en el proyecto no se establecían los mecanismos necesarios para comprobar el cumplimiento del principio de representación proporcional, ni los mecanismos de sanción para aquellos que no cumplieran con la disposición. Es decir, era otro proyecto vago e impreciso, que fue archivado.

Sin embargo, estos primeros intentos generaron grandes expectativas $y$ aspiraciones en las mujeres militantes de los distintos partidos políticos, lo que empezó a construir un importante movimiento, apoyado por varias instancias, como la Defensoría de la Mujer (Defensoría de los Habitantes), para que se reformara el Código Electoral de manera clara y precisa, con el objetivo de establecer el sistema de cuotas como un mecanismo de acatamiento obligatorio para los partidos políticos. Así, el 28 de noviembre de 1996 se reformó el artículo 60 del Código Electoral de forma tal que se obligaba a los partidos a incluir en sus estatutos los mecanismos necesarios para asegurar la participación de las mujeres en un porcentaje del $40 \%$ en la estructura partidaria, en las listas para los puestos de elección popular y en las 
delegaciones a las diferentes asambleas de los partidos (Bolaños, 2006).

Estas reformas al Código Electoral sentaron las bases de un acceso paritario de las mujeres a los puestos de poder y representaron un avance significativo en las normas legales tendientes a favorecer la equidad en la participación política. Asimismo, la discusión nacional sobre estas reformas permitió evidenciar todos los mitos existentes relativos a la participación política de las mujeres $y$ a poner en la palestra pública las voces de las mujeres que aspiraban a ocupar puestos de representación popular, pero que se habían visto impedidas por los diferentes mecanismos perversos de los pactos y arreglos patriarcales que operan al interior de las estructuras partidarias.

A pesar del avance que significó la aprobación de estas normas, en las elecciones nacionales de 1998 quedó en evidencia la gran distancia que existe entre la igualdad formal y la igualdad real. Se evidenció también la gran cantidad de estratagemas a las que pueden recurrir los partidos políticos cuando no existe una verdadera voluntad de garantizar la igualdad y la equidad a las mujeres. En esta ocasión, el principal problema se derivó de que la norma no había explicitado que el $40 \%$ de representación de las mujeres debía ser en puestos elegibles. Tampoco se había explicitado que no se aceptaría la inscripción de los partidos que no cumplieran con esta disposición.

Como resultado de una consulta hecha por el Instituto Nacional de las Mujeres, el Tribunal Electoral tuvo que informar a los partidos políticos de que no aceptaría la inscripción de ninguna papeleta que no estuviera conformada por un mínimo de un $40 \%$ de mujeres. Y aquí empezó la gran farsa. En las papeletas que cumplieron con la disposición, todas las mujeres fueron colocadas en los lugares finales de las listas, en puestos sin ninguna opción de elección. Se aceptó la inscripción de papeletas que no cumplían con la cuota porque los partidos alegaron que no habían encontrado suficientes mujeres que quisieran participar. Algunos partidos recurrieron al truco de sumar a todas las mujeres a escala nacional, sin considerar las papeletas por separado para cada municipio o provincia. En algunas papeletas ni siquiera colocaron a una sola mujer. De hecho, de los 23 partidos que presentaron candidaturas para la Asamblea Legislativa en las elecciones de 1998, 15 no cumplieron con la cuota (Torres, 2001). Fue entonces evidente que los partidos habían incluido a las mujeres en el viaje, pero no las querían en la llegada.

La experiencia de las elecciones de 1998 mostró claramente que si la normativa sobre cuotas no es explícita y estricta, la tradicional cultura política de los partidos, que está basada en una distribución masculina del poder $y$ el prestigio, va a fomentar el desarrollo de estrategias de mantenimiento y recuperación del poder, que funcionan en contra de la implementación del sistema de cuotas. En ese sentido, en 1998 hubo un avance significativo ya que las mujeres llegaron a representar el 19\% en el Congreso y el $30 \%$ en los gobiernos municipales (frente al 15\% y $12 \%$ respectivamente en las elecciones anteriores), la resistencia que generó la aplicación de este mecanismo al interior de los partidos políticos y la necesidad de reglas claras, explícitas y obligatorias quedaron en evidencia en esta elección.

El análisis de la puesta en práctica de la normativa en las elecciones nacionales de 1998 permitió construir las argumentaciones para solicitar al Tribunal Supremo de Elecciones la revisión de lo normado en relación con las cuotas. Después del amplio trabajo de cabildeo por parte de las organizaciones de mujeres y de la Defensoría de la Mujer, la solicitud de revisión fue presentada por Gloria Valerín, Ministra de la Condición de la Mujer y apoyada por Olga Nidia Fallas, magistrada del TSE. Como resultado, en la Resolución 1863 del 23 de setiembre de 1999, el Tribunal Electoral, interpretando el "espíritu" de la reforma de 1996, dispuso que:

$\diamond \quad$ El 40\% de participación de las mujeres en las papeletas debe ser en puestos elegibles.

$\diamond \quad$ El 40\% de cuota femenina debe respetarse en cada asamblea distrital, cantonal y provincial, $y$ no en forma global.

$\diamond \quad$ Cada partido tiene la obligación de incorporar en sus estatutos los ajustes nece- 
sarios para garantizar efectivamente la participación de las mujeres en la forma $y$ porcentajes dispuestos.

$\diamond \quad$ El Registro Civil no inscribirá las listas de candidatos que no se ajusten a estos parámetros.

$\diamond \quad$ El Registro Civil tampoco acreditará las reformas estatutarias ni las actas de las asambleas cuando se determine que no se cumplió con lo establecido.

En una resolución posterior (2837), del 12 de diciembre de 1999, a raíz de la consulta de varios partidos políticos que querían encontrar alguna forma de saltarse la norma, al alegar que no habían entendido el concepto de "puesto elegible", el Tribunal Electoral aclara lo siguiente:

Debe entenderse por puesto elegible... aquel que se asigna a una persona con posibilidades reales de ser electa $y$ por ello debe ser considerado individualmente en la conformación de las papeletas por cada provincia. Los partidos están obligados a implementar el sistema de cuotas de participación femenina $y$ debe- rán considerar que el porcentaje del $40 \%$ es un mínimo que, como tal puede incrementarse a favor de esa representación, pero no disminuirse.

El partido político está obligado a fomentar una cultura democrática y participativa que haga posible la incorporación de las mujeres.

Una vez que las reglas, procedimientos $y$ sanciones estuvieron totalmente claras, entonces se pudieron empezar a ver los efectos sustantivos del sistema de cuotas, ya que como se aprecia en los siguientes gráficos, en las elecciones del 2002 la representación de mujeres en la Asamblea Legislativa alcanzó el 35\% - convirtiendo al Congreso costarricense en el órgano con mayor representación de mujeres en América Latina hasta ese momento- $y$ en los municipios el porcentaje se incrementó a $47 \%$, casi llegando a la paridad (Bolaños, 2006). Durante las últimas dos elecciones de los años 2006 y 2010, la participación de las mujeres en la Asamblea Legislativa se incrementó al 38,5\% y en los municipios se mantuvo en valores cercanos al $45 \%$.

GRÁFICO 1

COSTA RICA: MUJERES REGIDORAS EN PUESTOS EN PROPIEDAD PERÍODO 1953-2006 NÚMEROS ABSOLUTOS

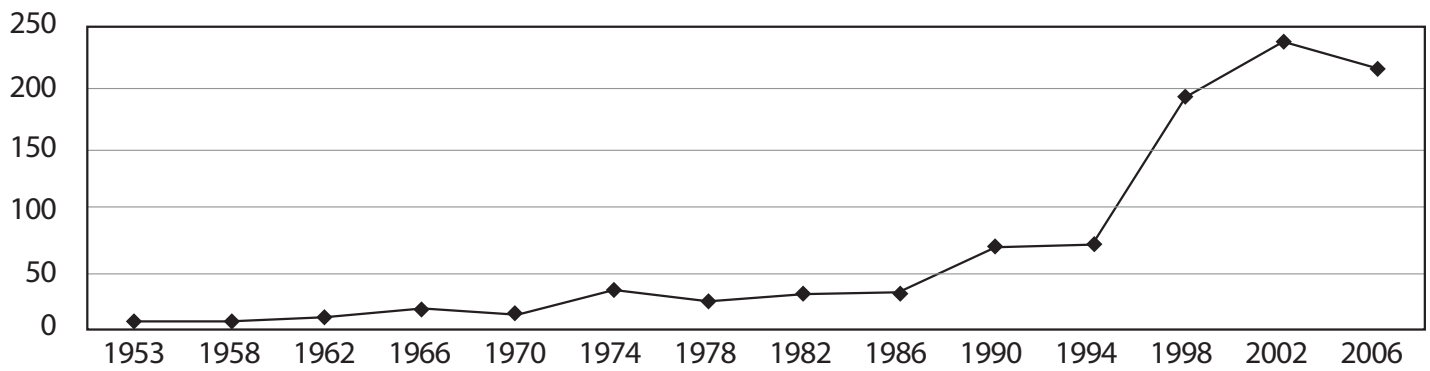

Fuente: Sistema de Indicadores de Género (SIEG) - Instituto Nacional de las Mujeres (INAMU). 
GRÁFICO 2

COSTA RICA: PARTICIPACIÓN FEMENINA EN LA ASAMBLEA LEGISLATIVA, 1982-2010 (PORCENTAJES)

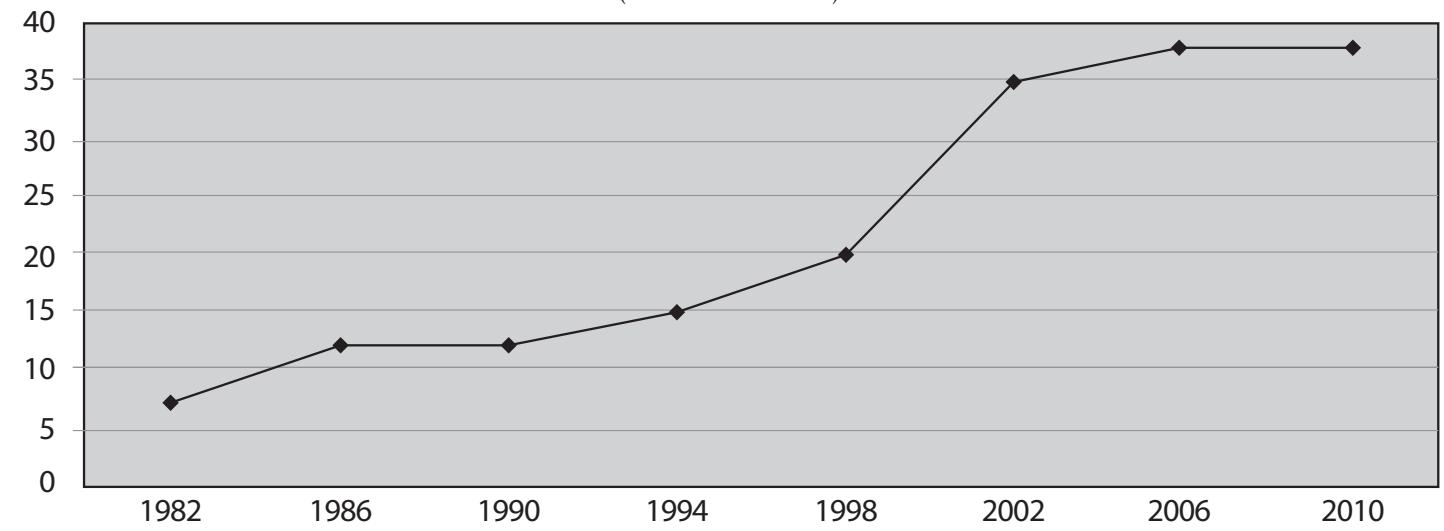

Fuente: Sistema de Indicadores de Género (SIEG) - Instituto Nacional de las Mujeres (INAMU).

La importancia de la utilización del mecanismo de las cuotas, con la obligatoriedad de colocar a un porcentaje de las mujeres en puestos elegibles, se ve reflejada no solo en el significativo incremento de la participación descriptiva de éestas en el Congreso y en los gobiernos locales, sino también cuando, por contraste, se analizan los espacios $y$ puestos donde no se impone el mandato legal de la cuota. En estos puestos, como la Corte Suprema de Justicia, las instituciones autónomas, el Servicio Exterior y las alcaldías, las mujeres continúan representando una franca minoría. Por ejemplo, para el año 2010, las mujeres solo ocupan el $11 \%$ de las alcaldías del país y representan el $30 \%$ de quienes integran la Corte Suprema de Justicia.

\section{AVANCES Y RETROCESOS HACIA LA EQUIDAD Y LA PARIDAD}

Ahora bien, como ejemplo de cómo los mecanismos creados por el movimiento feminista pueden también revertirse en su contra, vale la pena mencionar el caso del Partido Nueva Liga Feminista que participó en las elecciones de 2006. Cuando este partido presentó su inscripción, que pretendía llevar candidatas y una agenda abiertamente feminista al Congreso, esta fue inicialmente rechazada ya que el Registro Civil argumentó que entre sus listas no habían completado la cuota de hombres, es decir, el $40 \%$.

Finalmente, después de una ardua pelea, el Tribunal Supremo de Elecciones tomó una importante decisión que ayudó a clarificar no solo la legitimidad de la Nueva Liga Feminista y su posibilidad de inscribirse como partido político, a pesar de no contar con "suficientes" hombres en sus nóminas, sino también el sistema mismo de las cuotas para las mujeres, al crear jurisprudencia que especificaba aún más los mecanismos y ahondaba en su justificación como medida compensatoria. En esa ocasión el Tribunal Electoral declaró (Resolución 2096-E-2005 del 31 de agosto del 2005):

El $40 \%$ de la participación previsto en la normativa electoral es un mínimo y no un máximo... El desarrollo normativo de la cuota de participación de la mujer... es el reconocimiento del legislador de que, no obstante estar garantizado el principio de igualdad en la Constitución Política y en los diversos instrumentos sobre Derechos Humanos ratificados en el país, ha existido históricamente una desigualdad entre hombres y mujeres en el ámbito electoral que debe ser paliada con acciones afir- 
mativas, para evitar esa discriminación. La protección especial que se da en el Código Electoral a la mujer... no puede entenderse que comprende también al hombre, ya que sería admitir que éstos han sido igualmente discriminados, cuando lo cierto es que en las actividades político-electorales, los hombres se encuentran en condición ventajosa respecto a las mujeres...

Por otra parte, las discusiones sobre la necesidad de un nuevo Código Electoral, que se iniciaron desde la década de los años 90, sirvieron como un aliciente para que el movimiento de mujeres empezara a imaginar la posibilidad de incorporar el concepto de paridad en las nuevas regulaciones electorales. Además, la creación del Partido Acción Ciudadana (PAC) en el año 2000, que voluntariamente incluyó en sus reglamentos internos las nociones de paridad y alternancia por sexo en las listas electorales, abrió expectativas en las mujeres de otros partidos y reforzó la idea de que esta no era una meta imposible de alcanzar.

Finalmente, como resultado de las acciones emprendidas por el Instituto Nacional de las Mujeres, la Defensoría de la Mujer, algunas organizaciones feministas, legisladoras $y$ legisladores de diferentes partidos políticos ${ }^{3} y$ el mismo Tribunal Supremo de Elecciones, el proceso tendiente a garantizar la participación igualitaria de las mujeres en los puestos de representación popular tuvo un importante avance en el año 2009, con la aprobación del nuevo Código Electoral, que entrará a regir en su totalidad para las elecciones nacionales del 2014. Después de casi una década de acalorados debates y de una fuerte oposición por parte del Movimiento Libertario y de otros grupos, en el nuevo Código Electoral, aprobado en setiembre

3 Desde el año 2002, diputados y diputadas de diferentes partidos políticos, incluyendo a Emilia Rodríguez, Margarita Penón y Rodrigo Alberto Carazo (Partido Acción Ciudadana), Kyra de la Rosa (Liberación Nacional) y Gloria Valerín (Unidad Social Cristiana) empezaron a presentar mociones con el fin de incluir los principios de paridad y alternancia por sexo en el nuevo Código Electoral. de 2009 (Ley 8765), se define la participación política de hombres y mujeres en Costa Rica como un derecho humano al amparo de los principios de igualdad $y$ no discriminación, y se establecen de forma obligatoria la paridad y la alternancia por sexo.

Sobre la paridad y la alternancia por sexo, el nuevo Código Electoral, en su artículo 2, estipula:

La participación se regirá por el principio de paridad que implica que todas las delegaciones, las nóminas y los demás órganos pares estarán integrados por un cincuenta por ciento (50\%) de mujeres $y$ un cincuenta por ciento $(50 \%)$ de hombres, $y$ en delegaciones, nóminas $u$ órganos impares la diferencia entre el total de hombres y mujeres no podrá ser superior a uno.

Todas las nóminas de elección utilizarán el mecanismo de alternancia por sexo (mujer-hombre u hombre-mujer), en forma tal que dos personas del mismo sexo no puedan estar en forma consecutiva en la nómina.

El nuevo Código Electoral también estableció claramente que los estatutos de los partidos políticos deben contener las normas y los mecanismos que aseguren los principios de igualdad, equidad por género, no discriminación y paridad, tanto en la estructura partidaria como en las papeletas de elección popular. Asimismo, se incluyeron sanciones a los partidos que no cumplan con estos principios, incluyendo el rechazo o cancelación de su inscripción y la prohibición para participar en los procesos electorales.

Finalmente, se autorizó la contribución estatal, según una serie de disposiciones constitucionales, para que los partidos políticos desarrollen capacitación en forma permanente $y$ paritaria, tanto a hombres como a mujeres, con el objetivo de formar $y$ promover el conocimiento de los derechos humanos, la igualdad de géneros, incentivar los liderazgos, la participación política, el 
empoderamiento, la postulación y el ejercicio de puestos de decisión, entre otros.

Con estas reformas al Código Electoral, Costa Rica se convierte en uno de los primeros países de América Latina en establecer como norma legal obligatoria la paridad de género en materia de representación política. Es evidente que para las elecciones del 2014 esto implicará un cambio importante en el rostro de la política. Sin embargo, después de casi una década de aplicación progresiva del sistema de cuotas, el mecanismo también empieza a mostrar sus limitaciones para conseguir algunos de sus objetivos fundamentales, entre otros, la redistribución real del poder entre los géneros, la representación sustantiva de las mujeres $y$ la construcción de una sociedad más justa e igualitaria.

\section{RESULTADOS DE LA APLICACIÓN DEL SISTEMA DE CUOTAS EN COSTA RICA}

La primera lección aprendida sobre la aplicación de la normativa electoral sobre cuotas en Costa Rica es que este sistema solamente funciona si se definen unas normas y procedimientos claros, que incluyan sanciones para quienes no cumplen. Asimismo, es de suma importancia que exista un movimiento de mujeres fuerte y persistente, que empuje las reformas $y$ que se mantenga vigilante de su adecuado cumplimiento (Jones, 2004). En ese sentido, la efectividad de un sistema de cuotas para garantizar, al menos, la representación descriptiva de las mujeres depende de cinco factores:

1. Que sea parte de la legislación electoral nacional.

2. Que exista un movimiento de mujeres con la capacidad de fiscalizar el correcto cumplimiento de la normativa.

3. Que exista claridad en cuanto a las mecanismos de aplicación.

4. Que sea obligatorio colocar la cuota correspondiente a las mujeres en posiciones elegibles.

5. Que el órgano electoral esté directamente involucrado en la garantía del cumplimiento del sistema y que existan san- ciones claramente definidas y aplicadas consistentemente cuando se presentan incumplimientos.

Por otra parte, al permitir una mayor participación de las mujeres en los espacios de toma de decisiones se cuestiona la forma desigual en la que se ha ejercido $y$ distribuido el poder, los sistemas de cuotas tienen incidencia fundamentalmente en la política electoral y no en otros aspectos más centrales y sustantivos de una verdadera política de justicia de género. En ese sentido, las cuotas le cambian la cara a la política, lo cual significa un avance significativo $y$ una modificación de la cultura política, pero no necesariamente permite el avance de una agenda feminista de transformación social.

En el caso de Costa Rica, es interesante anotar que el desarrollo de normativa para ampliar los derechos de las mujeres tuvo un punto álgido en el país entre 1994 y el 2000, período en el que se aprobaron y promovieron el mayor número de leyes y reformas en la materia. En particular, en ese período se ratificó la Convención Interamericana para prevenir, sancionar y erradicar la violencia contra las mujeres (Convención de Belèm do Pará) y se aprobó una gran cantidad de legislación y políticas públicas sobre hostigamiento sexual, violencia doméstica, pensiones alimentarias, uniones de hecho, niñez y adolescencia, explotación sexual comercial, derechos sexuales y derechos reproductivos, entre otras temáticas ${ }^{4}$. Curiosamente, la legislación sobre cuotas no estaba todavía en vigencia en ese momento,

$4 \quad$ En ese período se aprueba la siguiente legislación: Ley contra el Hostigamiento Sexual en el Empleo y la Docencia (1995), Ley de Regulación de la Unión de Hecho (1995), Ley Contra la Violencia Doméstica (1996), Ley de Pensiones Alimentarias (1996), Código de la Niñez y la Adolescencia (1997), Ley General de Protección a la Madre Adolescente (1997), Ley de Creación del Instituto Nacional de las Mujeres (1998), Ley Contra la Explotación Sexual de Menores (1999), Decreto de Creación de la Comisión Interinstitucional sobre Salud y Derechos Reproductivos y Sexuales (1999) y el Decreto que establece el Registro Obligatorio de la Violencia Intrafamiliar en la Caja Costarricense del Seguro Social (1999). 
por lo que la representación de mujeres en el Congreso era relativamente baja. De hecho, estos avances, incluyendo la legislación misma sobre cuotas, parecen responder, más bien, a una expansión del quehacer del movimiento feminista local, que se produjo durante la década de los años 90, y no tanto a una mayor presencia de mujeres en puestos de elección popular.

De forma contradictoria, a partir del año 2001, cuando ya está en vigencia la normativa sobre cuotas, así como las aclaraciones hechas por el Tribunal Electoral para precisar esa normativa, disminuye sensiblemente el ritmo de la aprobación de leyes y políticas públicas relativas a la igualdad y equidad de género. En ese sentido, el aumento significativo de mujeres en la Asamblea Legislativa no parece tener un efecto directo en la justicia social o de género. Además, no parecen existir diferencias significativas entre las mujeres de los diferentes partidos políticos. Las pocas mujeres diputadas que desde el año 2001 han propuesto una agenda de igualdad y equidad de género parecen responder más a sus propios intereses, a su historia personal y a sus relaciones previas con el movimiento feminista, que a una línea clara de partido.

Por ejemplo, en la pasada legislatura, con $38 \%$ de representación femenina, solo una de las diputadas, Ana Helena Chacón, del Partido Unidad Social Cristiana, llevó adelante y de forma abierta un programa de justicia y equidad de género. Lo anterior demuestra que la relación que se da entre aumentar la presencia de mujeres en los parlamentos y la aprobación de leyes para el avance de la igualdad y equidad de género no es automática ni directa. Como lo plantea Susan Franceschet (2008), siguiendo a Debra Dodson (2006):

Mientras estudios existentes demuestran que en general es más probable que las legisladoras den prioridad a los temas de género que sus colegas hombres, su mayor preocupación por los temas relacionados con la mujer no se traduce directamente en mejores resultados legislativos para la misma (2008: 69).
Desde la anterior perspectiva, aunque la existencia de una normativa sobre cuotas aumenta la posibilidad de que las mujeres lleguen a puestos de elección popular, no hay garantía sobre el tipo de mujeres que llegará a ocupar esos puestos, ni sobre su agenda política. En el caso de Costa Rica, los principales partidos han elegido a mujeres altamente disciplinadas y fieles a la línea partidaria, con relaciones cercanas - casi subordinadas - con los hombres importantes de esos partidos y poco dispuestas a desafiar el statu quo. De hecho, los tres partidos mayoritarios tradicionales de derecha o centro-derecha (Liberación Nacional, Unidad Social Cristiana $y$ Movimiento Libertario), han nombrado a mujeres como jefas de fracción en momentos políticos particularmente álgidos, $y$ han sido estas mujeres precisamente las encargadas de llevar adelante la agenda neoliberal de estos partidos, incluyendo la ratificación del Tratado de Libre Comercio con los Estados Unidos, que tuvo en confrontación social al pueblo de Costa Rica por casi dos años.

Por eso, como lo plantean algunas autoras críticas del sistema de cuotas, en la práctica, las cuotas y el neoliberalismo no son mutuamente excluyentes, sino más bien, son socios en la búsqueda de un nuevo orden mundial (Krook, 2008). En el contexto de un neoliberalismo global creciente pareciera entonces que las cuotas significan una concesión importante a las demandas del movimiento de mujeres, pero a la vez, se convierten en una promesa vacía al contribuir a ensanchar la brecha creciente entre empoderamiento político por un lado, y empoderamiento social y económico, por otro (Phillips, 1999).

En el caso particular de Costa Rica, la mayoría de las diputadas electas después de la aprobación del sistema de cuotas — con la excepción de las diputadas del Partido Acción Ciudadana-, han estado más dispuestas a aprobar leyes que transforman el carácter benefactor del estado nacional, que legislación para ampliar la justicia social. Por ejemplo, la mayoría de las mujeres diputadas de la legislatura anterior (2006-2010) votaron a favor 
del Tratado de Libre Comercio con los Estados Unidos y de sus leyes complementarias, las que entre otras cosas, abrieron los mercados de las telecomunicaciones y de los seguros en el país, instituciones insignes del estado de bienestar desde 1948. Sin embargo, la mayoría de las legisladoras no han estado dispuestas a apoyar los proyectos de ley relativos a los derechos sexuales y reproductivos, las uniones civiles entre personas del mismo sexo, la autonomía de los pueblos indígenas o la reforma constitucional para establecer la separación entre la Iglesia y el Estado.

Derivado de lo anterior, surge la pregunta de si es más importante tener en un puesto de decisión a una persona que comparta las visiones del mundo del feminismo, o a una mujer, independientemente de su posición política, ideológica o ética. En Costa Rica, en la legislatura pasada, una de las posiciones más claras y contundentes en defensa de la justicia y la equidad de género fue representada por el diputado José Merino del Río, del Partido Frente Amplio, un partido minoritario de izquierda, pero que sí maneja un discurso consciente sobre las desigualdades sociales $y$ sobre la necesidad de enfrentarlas en todas sus dimensiones, incluyendo la dimensión de género.

En ese sentido, los sistemas de cuotas garantizan que más mujeres tengan acceso a puestos de representación popular, pero no garantizan la calidad de sus acciones ni el tipo de decisiones que van a tomar. Es decir, las cuotas fomentan la participación descriptiva de las mujeres, pero no necesariamente la sustantiva. Por esa razón, muchas feministas expresan sus dudas sobre las cuotas al argumentar, entre otras cosas, que estos mecanismos más bien ayudan a hacer avanzar los proyectos neoliberales, desmovilizan los movimientos de mujeres, resultan en la elección de mujeres conservadoras, promueven una visión estática de las mujeres como grupo y disminuyen su eficacia como actores políticos (Krook, 2008).

Sin embargo, otras feministas también argumentan que en una sociedad verdaderamente democrática, la participación de las mujeres en puestos de decisión y de poder debería entenderse como un derecho que no puede condicionarse a que estas luchen por los intereses de las mujeres. Desde esa perspectiva, los sistemas de cuotas representan un avance, pero deben ir necesariamente aparejados de otros procesos de empoderamiento y transformación social que realmente cuestionen el orden patriarcal; de lo contrario, solo se estarán provocando rupturas parciales en el sistema, pero no enfrentando sus bases, las que han generado no solo desigualdad, sino una sociedad éticamente insostenible.

La experiencia de Costa Rica demuestra que los sistemas de cuotas que incluyen la obligatoriedad de incorporar un porcentaje significativo de mujeres en puestos elegibles tiene una poderosa influencia sobre el número de mujeres que resultan electas; es decir, son un instrumento muy eficaz para incrementar la representación descriptiva de las mujeres. Sin embargo, aunque los sistemas de cuotas aseguran una mayor presencia de mujeres en los cuerpos legislativos, el sistema por sí solo no mejora la calidad de la democracia ya que no necesariamente expande la justicia social ni garantiza la representación de los intereses de las mujeres, como grupo históricamente oprimido y discriminado en la agenda pública.

Por esa razón, el objetivo del movimiento feminista no es y no puede ser simplemente el luchar por ampliar la representación de las mujeres en los puestos de elección popular o por mejores políticas o leyes. El objetivo del movimiento feminista debe ser continuar con la lucha iniciada hace más de 200 años por la construcción de una sociedad justa que les garantice a las mujeres $y$ a todos los grupos excluidos su autonomía, su bienestar y su integridad, $y$ un acceso igualitario al poder $y$ a los recursos de la sociedad.

\section{REFERENCIAS}

Badilla, Ana Elena. "Leading arguments against women's legal equality: highlights of a national debate". The Costa Rican Women's Movement. Editado por Ilse A. 
Leitinger. Pittsburg: The University of Pittsburg Press, 1997.

Bolaños, Arlette. "Las cuotas de participación política de la mujer en Costa Rica, 19962005”. Revista de Derecho Electoral. San José, Costa Rica: Tribunal Supremo de Elecciones, primer semestre, 2006.

Caul, Miki. "Political parties and the adoption of candidate gender quotas: a cross national analysis". The Journal of Politics 63 (4). November, 2001.

Camacho, Rosalía, Lara, Silvia y Serrano, Ester. Las cuotas mínimas de participación de las mujeres: mecanismo de acción afirmativa. San José, Costa Rica: Centro Nacional para el Desarrollo de la Mujer y la Familia, 1997.

Dahlerup, Drude. "El uso de cuotas para incrementar la representación política de la mujer". En: Mujeres en el Parlamento. Más allá de los números. Estocolmo, Suecia: Internacional IDEA. 1998.

Dodson, Debra L. The impact of women in Congress. Great Britain: Oxford University Press, 2006.

Franceschet, Susan. "¿Promueven las cuotas de género los intereses de las mujeres? El impacto de las cuotas en la representación sustantiva de las mujeres". En: Mujeres y politica: el impacto de las cuotas en América Latina. Editado por Marcela Ríos Tobar. Chile: Catalonia, 2008.

Freedman, Estelle (ed.). The essential feminist reader. New York: Modern Library, 2007.

Jones, Mark P. "Quota legislation and the election of women. Learning from the Costa Rican experience". The Journal of Politics 66 (4). November, 2004.

Klaussen, Jytte y Maier, Charles S. "Has liberalism failed women? Assuring equal representation in Europe and the United States". The American Political Science Review 96 (3). September, 2002.

Krook, Mona Lena. "La adopción e impacto de las leyes de cuotas de género: una perspectiva global". En: Mujeres y política: el impacto de las cuotas en América Latina. Editado por Marcela Ríos Tobar. Chile: Catalonia, 2008.

Maffía, Diana. ¿Le importan las mujeres a la democracia? 2004. En: <http://www. rimaweb.com.ar/> [consultado el 10/02/2010].

Marion Young, Iris. La justicia y la política de la diferencia. Valencia, España: Ediciones Cátedra, 2000.

Moller Okin, Susan. Justice, gender and the family. United States: Basic Books, 1989.

Pateman, Carole. El contrato sexual. Barcelona: Anthropos, 1995.

Phillips, Anne. Género y teoría democrática. México: PUEG-UNAM, 1996.

Phillips, Anne. Which equalities matter? Malden: Polity Press, 1999.

Ríos, Marcela (ed.). Mujer y política. El impacto de las cuotas de género en América Latina. Chile: Catalonia, 2008.

Ríos, Marcela y Villar, Andrés. Cuotas de género. Democracia y representación. Chile: FLACSO-IDEAS, 2006.

Sagot, Montserrat. "Introducción. De la exclusión a la participación política de las mujeres". En: Las mujeres y el poder. Editado por Linda Berrón. San José, Costa Rica: Editorial Mujeres, 1997.

Torres, Isabel. La aplicación de la cuota mínima de participación de las mujeres: ¿ficción o realidad? Un diagnóstico para Costa Rica. San José, Costa Rica: Fundación Arias para la Paz y el Progreso Humano, 2001. 
Tribunal Supremo de Elecciones. Sentencia nro. 1863. Setiembre 23. San José, Costa Rica: Tribunal Supremo de Elecciones, 1999.

Tribunal Supremo de Elecciones. Sentencia nro. 2837. Diciembre 12. San José, Costa Rica: Tribunal Supremo de Elecciones, 1999.
Tribunal Supremo de Elecciones. Sentencia nro.2096-E-2005. Agosto 31. San José, Costa Rica: Tribunal Supremo de Elecciones, 2005.

Fecha de ingreso: 20/09/2010 Fecha de aprobación: 25/04/2011 
